\title{
Performance of Cut-Off Wheels during Elastic Abrasive Cutting of Rotating Workpieces
}

\author{
Irina ALEKSANDROVA, Gancho GANEV, Hristo HRISTOV
}

\begin{abstract}
This paper presents a scheme for elastic abrasive cutting of rotating workpieces which ensures constant area of the instantaneous cross-section of the layer being cut-off by maintaining constant pressing force of the cut-off wheel to the rotating workpiece. Theoretical and experimental studies of the complex impact of compression force, cut-off wheel diameter and workpiece rotational frequency on tool wear and time per cut have been carried out. The optimum conditions for the process realization have been determined by compromise optimization. Mathematical models have been created for determining the number of cut-off cycles and the average duration of a cutoff cycle as parameters for evaluating the economic efficiency of the elastic abrasive cutting process.
\end{abstract}

Keywords: cut-off wheel; elastic abrasive cutting; time per cut; tool wear

\section{INTRODUCTION}

Abrasive cutting is widely used in industry owing to its high production rate, universality and economy $[1,2]$. The process is characterized by intensive wear of the abrasive cut-off tools, high temperature (above $1000^{\circ} \mathrm{C}$ ) in the cutting zone, spark formation, high noise level, danger of accidents, changes in the material microstructure in the surface layer and occurrence of thermal defects [3-9]. These shortcomings are related to the high cutting speed (over $50 \mathrm{~m} / \mathrm{s}$ ), continuous change in cutting conditions within a cut-off cycle, as well as to the unfavourable geometry of abrasive grains (negative rake angles). Therefore, the control of abrasive cutting process is possible mainly by changing the operating conditions. That requires complex study, modelling and optimization of the abrasive cutting process in order to enhance its efficiency and applicability.

Abrasive cutting of rotating workpieces can be done following different kinematical schemes (Fig. 1) [10], whereat the cut-off wheel performs two motions: main rotary motion at speed $V_{\mathrm{s}}$ and radial feed motion at speed $V_{\text {fp. }}$. The radial feed of the tool is provided either in a kinematical way by the cut-off machine as $V_{\mathrm{fp}}=$ const. (rigid abrasive cutting - diagrams 1 and 3, Fig. 1), or by maintaining constant force of compressing the wheel to the workpiece $\left(F=\right.$ const.), whereat $V_{\mathrm{fp}} \neq$ const. (elastic abrasive cutting - diagrams 2 and 4, Fig. 1). The workpiece is static or it rotates at constant rotational frequency $n_{\mathrm{w}}$.

The kinematical schemes of rigid abrasive cutting have been well studied [10]. The principal disadvantage of this method is the change in the power and heat loads of the abrasive tool within one cut-off cycle related to the change in the instantaneous cross-section area of the metal layer being cut.

The schematic diagrams of elastic abrasive cutting (diagrams 2 and 4, Fig. 1) are of interest. They ensure constant area of the instantaneous cross-section of the layer being cut, and consequently also stabilisation of the dynamic and thermal phenomena accompanying the cutting process [10]. This is a prerequisite for a more effective use of the cut-off wheel as well as for enhancing the quality of the machined surface.

During elastic abrasive cutting of a static workpiece (diagram 2, Fig. 1) the contact arc length $L$ and the thickness $h$ of the layer being cut vary, while the instantaneous cross-section area of the layer being cut remains constant. When the values of $L$ are large, regardless of the smaller thickness of the layer being cut, the chips separated during cutting cannot be held in the pores of the cut-off wheel. As a result the production rate of the process decreases and tool wear increases [11].

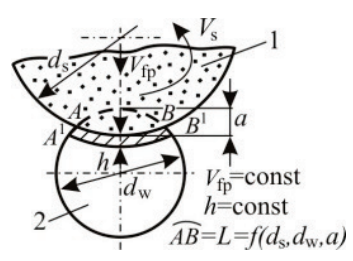

Diagram 1
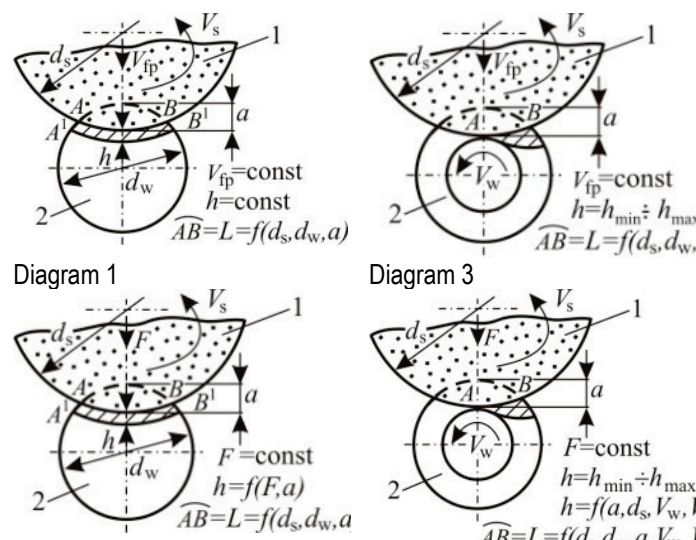
Diagram 3

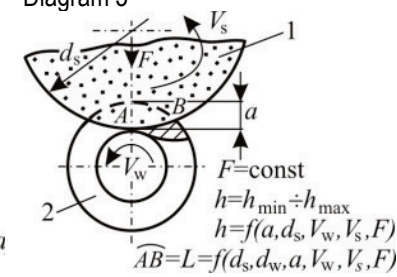

Diagram 2 Diagram 4

Figure 1 Schematic diagrams of abrasive cutting: 1 - cut-off wheel; 2 workpiece

The introduction of rotary motion of the workpiece (diagram 4, Fig. 1) leads to a decrease in the working stroke of the cut-off wheel, which ensures shorter time per cut and wear reduction of abrasive wheels. At the same time, the tool lifetime, process production rate, cutting forces, power rate and temperature will depend on the compression force $F$, workpiece rotational frequency $n_{\mathrm{w}}$, cut-off wheel diameter $d_{\mathrm{s}}$ and the type of material being machined. This makes this scheme for elastic abrasive cutting a topical object of study and optimization.

The objective of this paper is to present complex study and modelling of performance of cut-off wheels during elastic abrasive cutting with rotary motion of the workpiece and determining the optimum conditions for the process realization.

\section{THEORETICAL STUDY OF THE ELASTIC ABRASIVE PROCESS}

When the workpiece rotates at constant rotational frequency $n_{\mathrm{w}}$, the trajectory of a single abrasive grain during elastic cutting is a complicated curve whose 
character is shown in Fig. 2. Sectors 1-2, 3-4, 5-6, 7-8, ... of it correspond to the work of the cut-off wheel under a non-steady regime - regime under which cutting takes place when there is a radial feed motion at speed $V_{\mathrm{fp}} \neq$ const. and tangential feed at speed $V_{\text {fp }}=$ const. until the normal cutting force $F_{\mathrm{p}}$ becomes equal to the compression force $F$ of the cut-off wheel to the workpiece. When $F_{\mathrm{p}}=$ $F$, a steady-state regime is adopted and then $V_{\mathrm{fp}}=$ const. Cutting takes place only when there is tangential feed at speed $V_{\mathrm{ft}}$. By then the cut-off wheel has penetrated into the workpiece at a depth of $a_{1}$. Sectors $2-3,4-5,6-7,8-9, \ldots$ from the curve are obtained during operation of the cut-off wheel under steady-state regime and they are parts of concentric circumferences positioned at distances of $a_{2}<$ $a_{3}<a_{4}<\ldots<a_{k-1}<a_{k}$ ( $k$ is the number of the workpiece successive rotation). The difference in cutting depths $a_{1}, a_{2}$, $\ldots, a_{k}$ is due to a decrease in tangential feed as a result of a decrease in the workpiece diameter in the cutting zone.

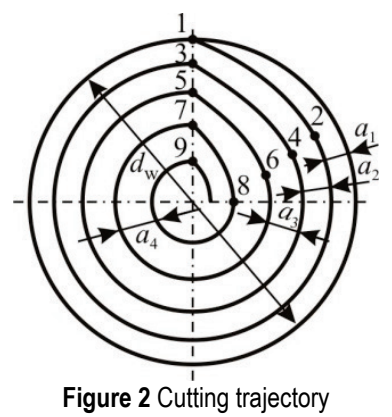

On the basis of kinematics analysis of elastic abrasive cutting process, the minimum theoretical value of workpiece rotational frequency $n_{\mathrm{w} \text { min, has been }}$ determined. At this frequency constant area $A$ of the instantaneous cross-section of the layer being cut is ensured and the cutting depth is $a=d_{\mathrm{w}} / 2$ [12]:

$n_{\mathrm{w} \min }=\frac{1,27 A n_{\mathrm{s}}}{d_{\mathrm{w}}^{2}} / \mathrm{min}^{-1}$,

where: $n_{\mathrm{s}}$ is the rotational frequency of the cut-off wheel; $d_{\mathrm{w}}$ is the workpiece diameter.

When workpiece rotational frequencies are $n_{\mathrm{w}}<n_{\mathrm{w} \text { min }}$ cutting is performed under a non-steady regime and when $n_{\mathrm{w}}$ decreases, the cutting depth increases up to $a=d_{\mathrm{w}}$. As a result, the friction forces along cut-off wheel front surfaces and the temperature in contact zones between the tool and workpiece will increase. All this is a prerequisite for wear growth and deterioration of cutting ability of the cut-off wheel.

When workpiece rotational frequencies are $n_{\mathrm{w}} \geq n_{\mathrm{w} \text { min }}$, cutting is performed according to the diagram in Fig. 2. When $n_{\mathrm{w}}$ increases, in order to meet the condition $A=$ const., the cutting depth and contact arc of the cut-off wheel with the workpiece decrease but the thickness of material layer being cut by one abrasive grain increases. As a result the abrasive grain load increases and there are prerequisites for hampered holding of chips in the cut-off wheel pores. At the same time the normal and tangential cutting forces ratio changes which results in deteriorating abrasive grains cutting abilities and decreasing the real area of the metal layer being cut. All this leads to intensification of wear of cut-off wheel, its cutting ability deterioration and to reduction in production rate.

The conducted analysis shows that workpiece rotational frequency has a great effect on the performance of the cut-off wheel. Rotational frequency $n_{\mathrm{w} \text { min }}$ exists, at which the cut-off wheel lifetime and the process production rate are maximum. The frequency magnitude depends on the area of the instantaneous cross-section of the layer being cut $A$, and therefore on the compression force of the cut-off wheel to the workpiece.

The kinematics of elastic abrasive cutting process involves also a great impact of compression force $F$ and cut-off wheel diameter $d_{\mathrm{s}}$ on the length of the contact arc of the tool with the workpiece, cutting depth and the thickness of the layer being cut and consequently on the tool lifetime and cutting ability. It has been found that an increase in compression force $F$ leads to an increase in contact arc length and cutting depth. This is a prerequisite for process production rate enhancement on one hand, but on the other - for an increase in abrasive tool wear. When the cut-off wheel diameter is shortened, the number of abrasive grains taking part in removing the cut layer for one tool revolution decreases, and as a result the thickness of the chip removed by one abrasive grain increases. Furthermore, when the cut-off wheel rotational frequency is constant, the decrease in diameter leads to a decrease in cutting speed which additionally deteriorates the cutting ability of abrasive grains. All this creates conditions for an increase in wear and deterioration of the cutting ability of the cut-off wheel [10-13].

The analysis carried out shows that an experimental study is necessary to determine the complex impact of workpiece rotational frequency, compression force and cut-off wheel diameter on the wheel performance during elastic abrasive cutting of rotating workpieces.

\section{PERFORMANCE OF CUT-OFF WHEELS DURING ELASTIC ABRASIVE CUTTING \\ 3.1 Experimental Study of the Performance of Cut-Off Wheels 3.1.1 Equipment, Materials and Methods}

The objective of the experiment is to find the correlation between the performance of cut-off wheels and operating conditions during elastic abrasive cutting. In this connection, compression force $F$, workpiece rotational frequency $n_{\mathrm{w}}$ and cut-off wheel diameter $d_{\mathrm{s}}$ have been chosen as control factors. Evaluation parameters for cut-off wheel performance are tool wear $\delta$ and time per cut $\tau$.

Cut-off wheel wear is determined as a mean value of the differences between tool diameters measured in two mutually perpendicular directions at the beginning and at the end of the respective cut-off cycle performed under specific cutting conditions. Time per cut corresponds to the duration of the respective cut-off cycle.

The general form of the models describing the relationship between the studied parameters (wear and time per cut) and the group of independent variables: factors $d_{\mathrm{s}}\left(x_{1}\right), F\left(x_{2}\right)$ and $n_{\mathrm{w}}\left(x_{3}\right)$, is:

$$
y_{g}=b_{0}+\sum_{i=1}^{3} b_{i} x_{i}+\sum_{i=1}^{3} b_{i i} x_{i}^{2}+\sum \sum_{i<j} b_{i j} x_{i} x_{j}
$$


where: $g=1,2 ; y_{1}=\delta(\mathrm{mm})$ and $y_{2}=\tau(\mathrm{s})$.

The form of the model has been chosen on the basis of the carried out theoretical study of the influence of conditions of elastic abrasive cutting on the cut-off wheel lifetime and cutting ability as the non-linear character of experimental dependencies $\delta=f\left(d_{\mathrm{s}}, F, n_{\mathrm{w}}\right)$ and $\tau=f\left(d_{\mathrm{s}}, F\right.$, $n_{\mathrm{w}}$ ) have been taken into account $[11,13]$.

For building the theoretical-experimental models (2), multi-factor designed experiments by orthogonal centralcomposition design have been conducted. The number of experiments is $N=2^{n}+2 n+1=15(n=2$ is the number of control factors). Three observations have been made for each experiment. The variation limits of control factors are presented in Tab. 1. They are determined on the basis of preliminary experimental studies of the performance of abrasive cut-off wheels and comprise the workpiece rotational frequency $n_{\mathrm{w} \text { min }}$, at which it is expected to obtain minimum wheel wear and minimum time per cut $[11,13]$.

Table 1 Factor levels in the experimental design

\begin{tabular}{|c|c|c|c|c|c|c|}
\hline \multirow{2}{*}{ Factors } & \multicolumn{5}{|c|}{ Factor levels } \\
\cline { 3 - 7 } & $-1,215$ & -1 & 0 & +1 & $+1,215$ \\
\hline$x_{1}$ & $d_{\mathrm{s}} / \mathrm{mm}$ & 120 & 125,3 & 150 & 174,7 & 180 \\
\hline$x_{2}$ & $F / \mathrm{daN}$ & 0,8 & 1 & 2 & 3 & 3,2 \\
\hline$x_{3}$ & $n_{\mathrm{w}} / \mathrm{min}^{-1}$ & 1 & 2 & 6 & 10 & 11 \\
\hline
\end{tabular}

The models are built by actually measured values of cut-off wheel wear and time per cut.

Experimental studies are conducted during counterdirectional cutting with cut-off wheels 41-180×22.2×3.0 A30RBF (EN 12413: 2007). The material being machined is steel C45 (1.0503 - EN 10277-2:2008) and it is in the form of cylindrical rods with diameter $d_{\mathrm{w}}=30 \mathrm{~mm}$.

To perform the elastic abrasive cutting process a special attachment has been designed (Fig. 3). It is fixed to the main carriage of a combination lathe, supplied with a device for stepless adjustment of workpiece rotational frequency $n_{\mathrm{w}}$. The attachment comprises an angle grinder which ensures constant rotational frequency of the cut-off wheel $\left(n_{\mathrm{s}}=8500 \mathrm{~min}^{-1}\right)$, and a unit for adjusting the amount of compression force $F$ of the cut-off wheel to the workpiece. An aspirator system is included to arrest, collect and remove the flying chips and sparks during abrasive cutting.

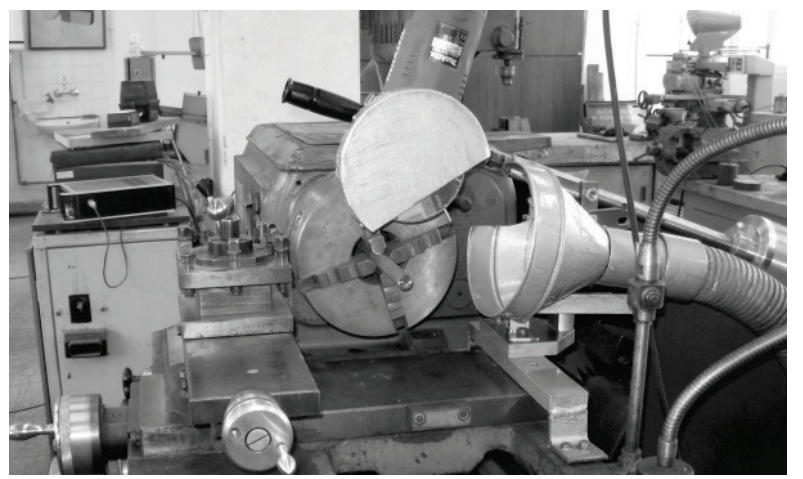

Figure 3 Workstand for elastic abrasive cutting

\subsubsection{Experimental Results}

The design of the experiments with the values of the studied response variables of the elastic abrasive cutting process are presented in Tab. 2. After statistical analysis of the experimental results and a transfer from coded to natural type of independent variables [14], theoreticalexperimental models of the cut-off wheel wear and time per cut have been built. Their general form is:

$$
\begin{aligned}
y_{g} & =B_{0, g}+B_{1, g} d_{\mathrm{s}}+B_{2, g} F+B_{3, g} n_{\mathrm{w}}+B_{33, g} n_{w}^{2}+ \\
& +B_{13, g} d_{\mathrm{s}} n_{\mathrm{w}}+B_{23, g} F n_{\mathrm{w}}
\end{aligned}
$$

Table 2 Design of the experiments and response variables of the elastic abrasive cutting process

\begin{tabular}{|c|c|c|c|c|c|}
\hline \multirow{2}{*}{ No } & \multicolumn{3}{|c|}{ Control factors } & \multicolumn{2}{c|}{ Response variables $y_{g e}$} \\
\cline { 2 - 6 } & $d_{\mathrm{s}} / \mathrm{mm}$ & $\begin{array}{c}\mathrm{F} / \\
\mathrm{daN}\end{array}$ & $\begin{array}{c}n_{\mathrm{w}} / \\
\mathrm{min}^{-1}\end{array}$ & $\begin{array}{c}\text { Tool wear } \\
\delta_{e} / \mathrm{mm}\end{array}$ & $\begin{array}{c}\text { Time per cut } \\
\tau_{e} / \mathrm{s}\end{array}$ \\
\hline 1 & 125,3 & 1 & 2 & 2,017 & 13,00 \\
\hline 2 & 174,7 & 1 & 2 & 1,550 & 11,67 \\
\hline 3 & 125,3 & 3 & 2 & 3,100 & 11,00 \\
\hline 4 & 174,7 & 3 & 2 & 2,650 & 9,67 \\
\hline 5 & 125,3 & 1 & 10 & 1,400 & 10,33 \\
\hline 6 & 174,7 & 1 & 10 & 0,667 & 9,00 \\
\hline 7 & 125,3 & 3 & 10 & 1,900 & 9,33 \\
\hline 8 & 174,7 & 3 & 10 & 1,167 & 8,00 \\
\hline 9 & 120 & 2 & 6 & 1,567 & 9,67 \\
\hline 10 & 180 & 2 & 6 & 0,833 & 8,00 \\
\hline 11 & 150 & 0,8 & 6 & 0,717 & 9,67 \\
\hline 12 & 150 & 3,2 & 6 & 1,650 & 8,00 \\
\hline 13 & 150 & 2 & 1 & 2,733 & 12,33 \\
\hline 14 & 150 & 2 & 11 & 1,467 & 9,67 \\
\hline 15 & 150 & 2 & 6 & 1,250 & 8,67 \\
\hline$\delta_{e}=\frac{1}{r} \sum_{l=1}^{r} \delta_{e, l} ; \tau_{e}=\frac{1}{r} \sum_{l=1}^{r} \tau_{e, l} ;$ & & \\
\hline$r=3$ & is the number of observations; $e=1 \div N ;$ & $N=15$ \\
\hline
\end{tabular}

The theoretical-experimental model (3) can be written in the form:

$$
y_{g}=p_{g}-q_{g} d_{\mathrm{s}},
$$

where: $p_{g}=B_{0, g}+B_{2, g} F+B_{3, g} n_{\mathrm{w}}+B_{23, g} F n_{\mathrm{w}}+B_{33, g} n_{\mathrm{w}}^{2}$ is a coefficient taking into account the effect of the workpiece rotational frequency and the compression force; $q_{g}=B_{1, g}+B_{13, g} n_{\mathrm{w}}$ is a coefficient taking into account the complex effect of the workpiece rotational frequency and the cut-off wheel diameter (Tab. 3).

Table 3 Coefficients of the theoretical-experimental models (4) and statistic characteristic of the models

\begin{tabular}{|c|c|c|c|c|}
\hline \multirow{2}{*}{$y_{g}$} & \multicolumn{3}{|c|}{ Coefficients } & \multicolumn{2}{c|}{$\begin{array}{c}\text { Fisher } \\
\text { criterion }\end{array}$} \\
\cline { 2 - 5 } & $p_{g}$ & $q_{g}$ & $\hat{F}_{\mathrm{R}, g}$ & $F_{\mathrm{T}, g}$ \\
\hline \multirow{2}{*}{$\delta$} & $\begin{array}{l}p_{1}=3,304+0,616 F- \\
-0,407 n_{\mathrm{w}}-0,037 F n_{\mathrm{w}}+ \\
+0,038 n_{\mathrm{w}}^{2}\end{array}$ & $\begin{array}{l}q_{1}=0,008+ \\
+0,0007 n_{\mathrm{w}}\end{array}$ & 0,3 & 2,27 \\
\hline \multirow{2}{*}{$\tau$} & $\begin{array}{l}p_{2}=20,015-1,105 F- \\
-1,508 n_{\mathrm{w}}+0,063 F n_{\mathrm{w}}+ \\
+0,093 n_{\mathrm{w}}^{2}\end{array}$ & $q_{2}=0,027$ & 0,04 & 2,18 \\
\hline
\end{tabular}

The constructed models (4) are adequate which is proved by comparing the empirical $\hat{F}_{\mathrm{R}, g}$ and tabular $F_{\mathrm{T}, g}$ 
values of Fisher criterion $\left(\hat{F}_{\mathrm{R}, g}<F_{\mathrm{T}, g}\right.$ at significance level $\alpha=0,05$, see Tab. 3). They describe with high accuracy the dependencies between the studied response variables and control factors. The values of the coefficient of multiple correlation are $\hat{R}_{g}=0,997 \div 0,998$.

The effect of cutting conditions on cut-off wheel wear and time per cut according to the created theoreticalexperimental models in coded form for the studied workpiece rotational frequencies is presented graphically in Figs. 4a and 4b. To study the surfaces determined by regression equations (4) in the field of the experiment at the same level as the output parameters, two-dimensional sections (Figs. $4 \mathrm{c}$ and $4 \mathrm{~d}$ ) have been built which are a family of lines.

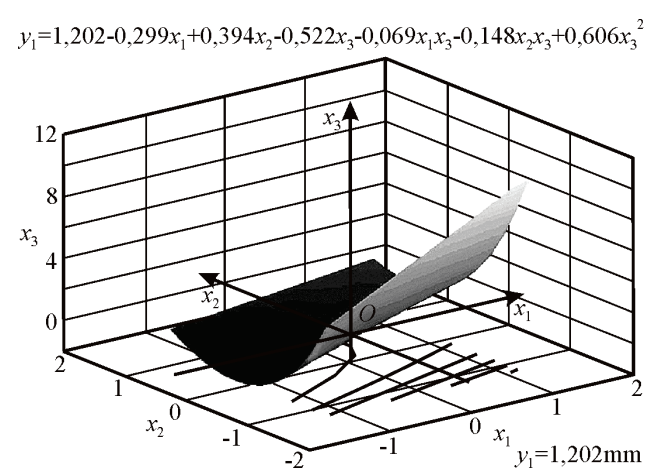

a)

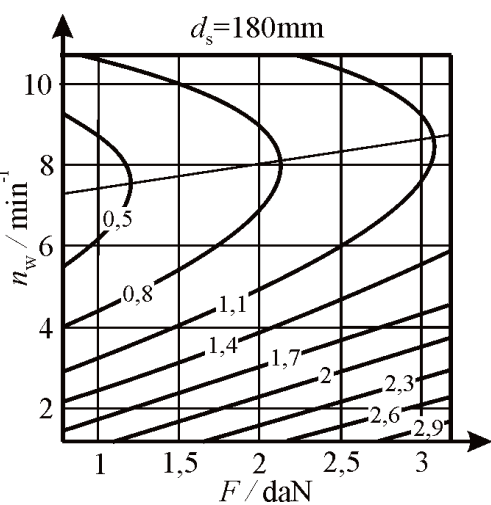

\subsubsection{Analysis of Results and Optimization}

The experimental results obtained confirm the theoretically determined character of impact of elastic abrasive cutting conditions on cut-off wheel wear and time per cut. The analysis of the created theoreticalexperimental models of tool wear $\delta$ and time per cut $\tau$ and the schematic representations (Fig. 4) plotted on their basis, allows the following conclusions to be drawn:

- When compression force in the studied range increases the time for elastic abrasive cutting decreases but cut-off wheel wear increases. This is related to increasing the contact arc length and cutting depth per tool revolution. The compression force has a greater effect on time per cut and this effect grows with increasing the workpiece rotational frequency.

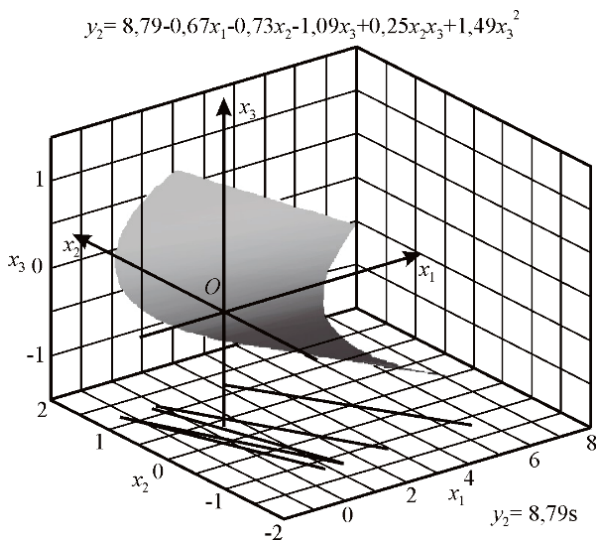

b)



d)

Figure 4 Effect of elastic abrasive cutting conditions on cut-off wheel wear $\delta / \mathrm{mm}(\mathrm{a}, \mathrm{c})$ and time per cut $\tau / \mathrm{s}(\mathrm{b}, \mathrm{d})$

- When the cut-off wheel diameter decreases, tool wear increases and the time for elastic abrasive cutting increases. This can be explained by a drop in cutting speed since cut-off wheel rotational frequency is constant, with decreasing the number of abrasive grains taking part in removing the layer being cut per revolution of the cut-off wheel and with increasing the thickness of the removed chip by one abrasive grain. All this causes an increase in load of abrasive grains and deteriorates their cutting ability and that naturally leads to an increase in time per cut and tool wear.

- Functional relationships (4) concerning wear and time per cut have minimums which are obtained respectively at rotational frequencies $n_{\mathrm{wo}}^{\prime}$ and $n_{\mathrm{wo}}^{\prime \prime}$ $\left(n_{\mathrm{wo}}^{\prime} \neq n_{\mathrm{wo}}^{\prime \prime}\right)$. The values of $n_{\mathrm{wo}}^{\prime}$ and $n_{\mathrm{wo}}^{\prime \prime}$ depend on the compression force of the cut-off wheel to the workpiece and on the tool diameter:

$n_{\mathrm{wo}}^{\prime}=5,3783+0,0093 d_{s}+0,4894 F$,

$n_{\mathrm{wo}}^{\prime \prime}=8,1486-0,3378 F$.

Compression force $F$ has a different effect by character on rotational frequencies $n_{\mathrm{wo}}^{\prime}$ and $n_{\mathrm{wo}}^{\prime \prime}$. With regard to this, when the tool diameter is one and the same, minimum wear and minimum time per cut are obtained for different values of the compression force and workpiece rotational frequency - $F=0,8 \mathrm{daN}, \quad n_{\mathrm{wo}}^{\prime}=7,44 \mathrm{~min}^{-1}$ and 
$F=3,2 \mathrm{daN}, n_{\mathrm{wo}}^{\prime \prime}=7,07 \mathrm{~min}^{-1}$. This requires carrying out compromise optimization for determining the optimum conditions for elastic abrasive cutting process. To this end, in the capacity of optimization parameter, the generalized utility function has been chosen determined in correspondence with the relationship [14]:

$$
\Phi=\frac{1}{2} \sum_{g=1}^{2} \frac{k_{g}\left(y_{g}-y_{g, \mathrm{u}}\right)}{y_{g \max }-y_{g \min }},
$$

where: $k_{g}$ is utility coefficient; $k_{g}-1$, when the reduction of $y_{g}$ is useful $\left(k_{1}=k_{2}=-1\right) ; y_{g, \mathrm{u}}$ is the least desirable result of parameter $y_{g}$, obtained in the range of the studied factor space: $y_{1, \mathrm{u}}=y_{1 \max } ; y_{2, \mathrm{u}}=y_{2 \max } ; y_{g \max }, y_{g \text { min }}$ are utility limits (maximum and minimum values of parameter $y_{g}$ - Tab. 2).

After substituting relationships (4) in Eq. (7) a mathematical model of the generalized utility function is obtained. It reflexes the complex effect of the control factors of the elastic abrasive cutting process:

$$
\begin{gathered}
\Phi=-0,7273+0,0043 d_{\mathrm{s}}-0,0185 F+0,2321 n_{\mathrm{w}}- \\
-0,0169 n_{\mathrm{w}}^{2}+0,0001 d_{\mathrm{s}} n_{\mathrm{w}}+0,0015 F n_{\mathrm{w}}
\end{gathered} .
$$

Compromise optimization is reduced to determining a combination of values of the selected control factors: workpiece rotational frequency, compression force and cut-off wheel diameter, when the generalized utility function has a maximum.

The maximum value of $\Phi=f\left(d_{\mathrm{s}}, F, n_{\mathrm{w}}\right)$ is obtained for workpiece rotational frequency $n_{\mathrm{wo}}\left(n_{\mathrm{wo}} \neq n_{\mathrm{wo}}^{\prime} \neq n_{\mathrm{wo}}^{\prime \prime}\right)$, whose value depends on the cut-off wheel diameter $\left(d_{\mathrm{s}}\right)$ and the compression force of the tool to workpiece $(F)$ :

$n_{\mathrm{wo}}=6,8669+0,0041 d_{\mathrm{s}}+0,0015 F$.

It can be seen that in the range of large diameters of the cut-off wheel, when compression force increases, workpiece rotational frequency $n_{\mathrm{wo}}$ rises, at which the maximum value of the generalized target function is obtained. This can be explained by an increase in the crosssection of the metal layer being cut per revolution of the cut-off wheel.

The combination of values of the cut-off wheel diameter, compression force and workpiece rotational frequency for which the maximum value of the generalized utility function $(\Phi=0,97)$ is obtained, is $d_{\mathrm{s}}=180 \mathrm{~mm}, F=$ $0,8 \mathrm{daN}$ and $n_{\mathrm{wo}}=7,61 \mathrm{~min}^{-1}$.

\subsection{Number of Operating Cycles and Average Duration of a Cut-Off Cycle}

In order to evaluate the economic efficiency of elastic abrasive cutting process it is necessary to determine the number of cut-off cycles, which the cut-off wheel can perform under specified cutting conditions as well as the average duration of one cut-off cycle.

During cutting, as a result of wear, the cut-off wheel diameter changes within the range from $d_{\mathrm{s} \max }=d_{s 0}$ (initial wheel diameter) up to $d_{\mathrm{s} \min }=d_{s, m}$ (wheel diameter after the last cut-off cycle) according to the relationships:

$$
\begin{aligned}
& d_{s_{1}}=d_{s_{0}}-\delta_{1}=\left(q_{1}+1\right) d_{s_{0}}-p_{1} ; \\
& d_{s_{2}}=d_{s_{1}}-\delta_{2}=\left(q_{1}+1\right)^{2} d_{s_{0}}-\left(q_{1}+1\right) p_{1}-p_{1} ; \\
& d_{\mathrm{s}, m}=d_{\mathrm{s},(m-1)}-\delta_{m}= \\
& =\left(q_{1}+1\right)^{m} d_{\mathrm{s}_{0}}-\left(q_{1}+1\right)^{m-1} p_{1}-\ldots . .-\left(q_{1}+1\right) p_{1}-p_{1},
\end{aligned}
$$

where: $p_{1}$ and $q_{1}$ are coefficients of the theoreticalexperimental model of cut-off wheel wear (4) (Tab. 3); $m$ is the number of cut-off cycles.

After transforming the diameter relationship (10), it is obtained:

$$
d_{\mathrm{s}, m}=\left(d_{s_{0}}-p_{1} / q_{1}\right)\left(q_{1}+1\right)^{m}+p_{1} / q_{1} .
$$

The relationship for determining the number of operating cycles $m$, performed by one cut-off wheel under specified cutting conditions obtained after solving Eq. (11), is:

$$
m=\frac{\ln \left[\left(d_{\mathrm{s}, m} q_{1}-p_{1}\right) /\left(d_{\mathrm{s}_{0}} q_{1}-p_{1}\right)\right]}{\ln \left(q_{1}+1\right)} .
$$

The average duration of a cut-off cycle $t_{\mathrm{c}}$ is determined by:

$t_{\mathrm{c}}=\frac{1}{m} \sum_{i=1}^{m} \tau_{i} / \mathrm{s}$

where $\tau_{1}, \tau_{2}, \ldots \ldots, \tau_{m-1}, \tau_{m}$ are durations of operating cycles performed by one cut-off wheel when its diameter has changed from $d_{\mathrm{s} \max }=d_{s 0}$ to $d_{\mathrm{s} \min }=d_{s, m}$. They are determined in accordance with the theoreticalexperimental model (4) and Tab. 3. Therefore:

$$
\sum_{i=1}^{m} \tau_{i}=p_{2} m-q_{2} \sum_{i=0}^{m-1} d_{\mathrm{s}, i}
$$

To determine $\sum_{i=0}^{m-1} d_{\mathrm{s}, i}$ relationships (10) are employed for changing the cut-off wheel diameter and after transformation it is obtained:

$$
\sum_{i=0}^{m-1} d_{\mathrm{s}, i}=\frac{p_{1} q_{1} m+\left[\left(q_{1}+1\right)^{m}-1\right]\left(q_{1} d_{\mathrm{s}_{0}}-p_{1}\right)}{q_{1}^{2}}
$$

Taking into account relationships (13), (14) and (15), the following model is obtained for the average duration of the cut-off cycle:

$t_{\mathrm{c}}=p_{2}-q_{2} \frac{p_{1} q_{1} m+\left[\left(q_{1}+1\right)^{m}-1\right]\left(q_{1} d_{\mathrm{s}_{0}}-p_{1}\right)}{m q_{1}^{2}}$. 
With the help of the created relationships (12) and (16) for the specified optimum conditions of elastic abrasive cutting of rotating workpieces of $\mathrm{C} 45$ steel with a diameter of $30 \mathrm{~mm}$ with cut-off wheels $41-180 \times 22.2 \times 3.0 \mathrm{~A} 30 \mathrm{RBF}$, the number of operating cycles and the average duration of one cut-off cycle: $m=101$ and $t_{\mathrm{c}}=9,2 \mathrm{~s}$ have been found.

\section{CONCLUSION}

On the basis of analysis of the advantages and disadvantages of various kinematical schemes of abrasive cutting process, a scheme of elastic abrasive cutting of rotating workpieces has been proposed in which the radial feed of the cut-off wheel is performed by a constant compressive force of the wheel to a rotating rod. This scheme ensures constant area of the instantaneous crosssection of the material layer being cut, stabilization of the dynamic and thermal phenomena accompanying the cutting process and reduction of the tool working stroke, and as a result - an increase in: the cut-off wheel lifetime, process production rate and the quality of the machined surfaces. To implement the scheme an experimental stand has been designed which enables the setting and adjustment of conditions for elastic abrasive cutting.

As a result of the conducted theoretical and experimental studies, the modelling and the optimization of the elastic abrasive cutting process of rotating workpieces the following results have been achieved:

(1) By analysing the kinematics of elastic abrasive cutting process, the effect of the workpiece rotational frequency, compression force and diameter of the cut-off wheel on the tool lifetime and cutting ability has been studied. It has been proved that there is a workpiece rotational frequency during cutting at which the cut-off wheel lifetime and process production rate are maximum, and the frequency magnitude depends on the compression force of the cut-off wheel to the workpiece.

(2) Experimental study of wear of cut-off wheels 41$180 \times 22.2 \times 3.0 \mathrm{~A} 30 \mathrm{RBF}$ and of the time per cutting rotating workpieces of $\mathrm{C} 45$ steel during elastic abrasive cutting has been carried out. Adequate theoretical-experimental models have been obtained reflecting the complex effect of compression force of cut-off wheel to the workpiece, workpiece rotational frequency and cut-off wheel diameter on the studied parameters. The created theoreticalexperimental models have been analyzed and it has been found that of all studied factors the influence the compression force on the performance of the cut-off wheels is the strongest, as with an increase in $F$ in the studied range the time for elastic abrasive cutting decreases but cut-off wheel wear increases. The impact rate of the compression force depends on the workpiece rotational frequency. It has been found that the minimum wear and minimum time per cut are obtained for different combinations of values of the compression force and workpiece rotational frequency, as follows: $F=0,8 \mathrm{daN}$, $n_{\mathrm{wo}}^{\prime}=7,44 \mathrm{~min}^{-1}$ and $F=3,2 \mathrm{daN}, n_{\mathrm{wo}}^{\prime \prime}=7,07 \mathrm{~min}^{-1}$.

(3) By compromise optimization the optimum conditions for performing elastic abrasive cutting process have been determined. In the capacity of optimization parameter, the generalized utility function has been chosen for which a mathematical model has been created which reflects the complex effect of the selected control factors of elastic abrasive cutting process. On the basis of the obtained results we can recommend the following combination of values of the cut-off wheel diameter, compression force and workpiece rotational frequency for which the maximum value of the generalized utility function $(\Phi=0,97)$ is obtained: $d_{\mathrm{s}}=180 \mathrm{~mm}, F=0,8 \mathrm{daN}$ and $n_{\mathrm{wo}}=7,61 \mathrm{~min}^{-1}$.

(4) Models have been built for determining the number of cut-off cycles which can be realized by the cut-off wheel under specific cutting conditions and for the average duration of a cut-off cycle. The number of cut-off cycles and the average duration of a cut-off cycle have been determined under optimum conditions during cutting workpieces of C45 steel with cut-off wheel 41$180 \times 22.2 \times 3.0$ A30RBF $-m=101$ and $\tau_{\mathrm{m}}=9,2 \mathrm{~s}$.

\section{REFERENCES}

[1] Nagasaka, K., Yoshida, T., Kita, Y., \& Hashimoto, F. (1987). Optimum combination of operating parameters in abrasive cut-off. International Journal of Machine Tool \& Manufacture, 27(2), 167-179. https://doi.org/10.1016/S0890-6955(87)80048-2

[2] Vologin, K. (2002). Selection of rational conditions for cutting workpieces of difficult to machine material with abrasive wheels (Extended abstract of PhD thesis). Moscow State Technological University "Stankin", Moscow. (in Russian)

[3] Kaczmarek, K. (2008). The effect of abrasive cutting on the temperature of grinding wheel and its relative efficiency. Archives of Civil and Mechanical Engineering, 8(2), 81-91. https://doi.org/10.1016/S1644-9665(12)60195-2

[4] Kaczmarek, K. (2011). Using a thermovision method for measuring temperatures of a workpiece during abrasive cutoff operation. Advance in Manufacturing science and Technology, 35(4), 85-95. Retrieved from http://advancesmst.prz.edu.pl/pdfy/08a-Kaczmarek-Jozefn.pdf

[5] Levchenko, E. (2010). Theoretical study of features of operation of cut-off wheel side surfaces during abrasive cutting of pipes. VesnikSevNTU, 107, 114-117. (in Russian)

[6] Neugebauer, R., Hess, K.-U., Gleich, S., \& Pop, S. (2005). Reducing Tool Wear in Abrasive Cutting. International Journal of Machine Tools \& Manufacture, 45(10), 11201123. https://doi.org/10.1016/j.jimachtools.2005.01.002

[7] Luo, S. Y., Tsai, Y. Y., \& Chen, C. H. (2006). Studies on cut-off grinding of BK7 optical glass using thin diamond wheels. Journal of Materials Processing Technology, 173(3), 321-329. https://doi.org/10.1016/j.jmatprotec.2005.11.036

[8] Sahu, P. \& Sagar, R. (2006). Development of abrasive cutoff wheel having side grooves. International Journal of Advanced Manufacturing Technology, 31(1-2), 37-40. https://doi.org/10.1007/s00170-005-0138-2

[9] Yoshida, T., Nagasaka, K., Kita, Y., \& Hashimoto, F. (1986). Identification of a grinding wheel wear equation of the abrasive cut-off by the modified GMDH. International Journal of Machine Tool Design and Research, 26(3), 283292. https://doi.org/10.1016/0020-7357(86)90006-5

[10] Nenkov, N., Aleksandrova, I., \& Ganev, G. (1999). Methods of abrasive cutting of workpieces. Mashinostroene, 5-6, 3840. (in Bulgarian)

[11] Ganev, G., Aleksandrova, I. \& Hristov, H. (2012) Wear and lifetime of abrasive tools during elastic abrasive cutting. Machine Building and Electrical Engineering, 10-11, 20-25. (in Bulgarian) 
[12] Aleksandrova, I., Hristov, H., \& Ganev, G. (2011). Dynamic and Technological Characteristics of the Process Elastic Abrasive Cutting of Rotating Workpieces. Journal of the Technical University Sofia, Branch Plovdiv, 16, 123-128. (in Bulgarian)

[13] Ganev, G., Aleksandrova, I., \& Hristov, H. (2012). Study and modelling of time for elastic abrasive cutting of rotating workpieces. Machine Building and Electrical Engineering, 12, 26-31. (in Bulgarian)

[14] Aleksandrov, A. \& Aleksandrova, I. (2012). Theory of experiment. Gabrovo, Ex-Press. (in Bulgarian)

\section{Contact information:}

Irina ALEKSANDROVA, Prof., PhD

(Corresponding author)

Technical University of Gabrovo

Department of Mechanical Engineering Equipment and Technologies

4 H. Dimitar St, Gabrovo 5300, Republic of Bulgaria

E-mail: irina@tugab.bg

\section{Gancho GANEV, PhD}

Ideal Standard - Vidima

53 M. Popov St, Sevlievo 5400, Republic of Bulgaria

E-mail: ganevg1971@abv.bg

Hristo HRISTOV, Assoc. Prof., PhD

Technical University of Gabrovo,

Department of Mechanical Engineering Equipment and Technologies,

4 H. Dimitar St, Gabrovo 5300, Republic of Bulgaria

E-mail: cristof@tugab.bg 